\title{
Sarcoma de kaposi pulmonar en un paciente con sida, reporte de caso
}

\section{kaposi pulmonary sarcoma in a patient with aids, case report}

DOI: $10.46932 /$ sfjdv2n2-135

Received in: March 1st, 2021

Accepted in: May 30th, 2021

Md. Román Zambrano María del Cisne

Médica

Residente de Medicina Interna del Hospital IESS General Machala

E-mail: cisne-1991@ hotmail.com

Md. López Bravo Marcela Tatiana

Médica

Residente de Medicina Interna del Hospital IESS General Machala

E-mail: marcelalopezbravo@outlook.com

Md. Mejía Suarez Eliana Estefanía

Médica

Residente de Pediatría del Hospital IESS General Machala

E-mail: elianam-17@hotmail.com

Md. Ortega García Ivonne del Rocío

Médica

Residente de Medicina Interna del Hospital IESS General Machala

E-mail: ivon_131@hotmail.com

*Md. Peña Armijos Madeleyne Victoria

Médica

Residente de Medicina Interna del Hospital IESS General Machala

E-mail: madeley2823@gmail.com

\section{Md. Ortiz Cumbe José Adrián}

Médico

Residente de Traumatología del Hospital Teófilo Davila

E-mail: jaortiz93@gmail.com

Dr. Alvarado Maldonado Danny Ismael,

Médico Especialista en Neumología

Hospital General IESS Machala

E-mail: d.alvaradomaldonado@gmail.com

Dr. Valarezo Concha Cledy Alcides

Médico Especialista en Infectología

Hospital General IESS Machala

E-mail: cledyconcha@ hotmail.com 


\section{RESUMEN}

El Sarcoma de Kaposi (KS) es una neoplasia maligna del endotelio vascular que se asocia con infección por el Herpes Virus tipo 8 (HHV-8) (Lodi S, 2010). La mayoría de casos de KS que presentan afectación pulmonar se acompañan con presentación mucocutánea (80 - 90\% de casos) (Haramati LB, 2000) .Es más frecuente en el sexo masculino, y en pacientes inmunocomprometidos (Onak Kandemir N, 2013), (Jiménez F, 2011)

El objetivo de nuestra investigación fue realizar un reporte de caso de Sarcoma de Kaposi Pulmonar en un paciente con SIDA, utilizando como Metodología: la historia clínica completa del paciente, los estudios complementarios, incluyendo los invasivos como la fibrobroncoscopia, Biopsia de piel, que fueron de gran importancia para llegar a la etiología de la enfermedad.

A continuación, se presenta el caso clínico de un paciente masculino de 22 años de reciente diagnóstico de SIDA que presentó lesiones cutáneas induradas papulosas de coloración rojo vinosas acompañado de sintomatología respiratoria.

Palabras claves: Sarcoma de Kaposi (SK) pulmonar, SIDA, HIV, Herpes Virus tipo 8

\section{SUMMARY}

Kaposi's Sarcoma (KS) is a malignant neoplasm of the vascular endothelium that is associated with Herpes Virus type 8 (HHV-8) infection (Lodi S, 2010). The majority of cases of KS that present with pulmonary involvement are accompanied by mucocutaneous presentation (80 - 90\% of cases) (Haramati LB, 2000). It is more frequent in males, and in immunocompromised patients (Onak Kandemir N, 2013), (Jiménez F, 2011)

The objective of our research was to carry out a case report of Pulmonary Kaposi Sarcoma in a patient with AIDS, using as Methodology: the complete clinical history of the patient, complementary studies, including invasive ones such as bronchoscopy, skin biopsy, which were of great importance to arrive at the etiology of the disease

The following is the clinical case of a 22 -year-old male patient with a recent diagnosis of AIDS who presented papulous indurated dermal lesions of red wine color accompanied by respiratory symptoms.

Keywords: Pulmonary Kaposi (SK) sarcoma, AIDS, HIV, Herpes Virus type 8

\section{INTRODUCCIÓN:}

El Sarcoma de Kapossi fue descrito originalmente por Moritz Kaposi, dermatólogo húngaro en la Universidad de Viena en 1872, y es conocida como una de las enfermedades definitorias del SIDA desde la década de 1980 (Onak Kandemir N, 2013).

Este tumor presenta una gran agresividad porque su afectación es multisistémica y de mal pronóstico, constituye el elemento diagnóstico en aproximadamente 9-10\% de los casos de SIDA (Jiménez F, 2011). Se reporta en 15\% de la población mundial afectada con SIDA. Hasta la epidemia de SIDA este proceso neoplásico era raro. Sin embargo, a partir de 1980 el sarcoma de Kaposi se convirtió en la neoplasia más frecuente en los pacientes infectados por el VIH. (JA., 2015)

Sarcoma de Kaposi epidémico o asociado a virus de inmunodeficiencia humana (VIH): Es hasta 20 veces más frecuente en varones homosexuales con VIH. Tiene predilección por la región cefálica 
(párpados, nariz y orejas). La afectación de mucosas es frecuente, sobre todo a nivel oral, y puede ser la manifestación inicial de la enfermedad en un $15 \%$ de estos casos. Estos pacientes pueden presentar afectación extracutáneas: gastrointestinal, pulmonar y ganglionar. (Enríquez., enero-marzo 2013)

\subsection{SARCOMA DE KAPOSI PULMONAR}

Es una variante muy infrecuente, de mal pronóstico, tiempo mediano de supervivencia para las personas diagnosticadas con sarcoma de Kaposi pulmonar fue sólo de 19 meses. La supervivencia global a cinco años fue significativamente más pobre entre personas con sarcoma de Kaposi pulmonar que entre aquellos con sarcoma de Kaposi no pulmonar (49\% frente a 82\%, p<0,0001) (Palmieri C. et al., 2006)

Manifestaciones clínicas: No hay sintomatología determinante para distinguir al KS pulmonar de otras patologías respiratorias.

Por lo general se caracteriza por disnea, hipoxemia, tos seca desarrollada en pocas semanas. También puede presentarse con hemoptisis, fiebre, astenia e insuficiencia respiratoria, sibilancias, en algunos casos el KS endobronquial puede cursar asintomático

Puede verse afectado el parénquima pulmonar, las vías respiratorias, pleura y/o conductos linfáticos. El derrame pleural puede afectar hasta 2/3 de pacientes con KS intraparenquimatoso, y ocasionalmente puede ocurrir como una manifestación aislada. (Kevin R Flaherty, 2019), (P Cacopardo B, 2015), (Joshi M, 2006)

\subsection{TOMOGRAFÍA DE TÓRAX}

Dos hallazgos son sugestivos de KS parenquimatoso: densidades hiliares que se extienden dentro del parénquima a lo largo de las vías perivasculares o peribronquiales y un patrón septal o nodular característico con derrames pleurales concomitantes (Nathan S, 1990), (Hoskote SS, 2012).

Broncoscopia y lavado broncoalveolar (BAL): Las lesiones son generalmente planas, maculares o papulares, de color violáceo o rojo brillante, los sitios de aparición más frecuente son las vías aéreas inferiores y con menos frecuencia en tráquea. (Yoo DJ, 2005)

Patología: las características microscopias pueden incluir angiogénesis, inflamación y proliferación de células fusiformes. Las células del huso manchan constantemente para CD34 y CD31 y son negativas para el factor 8. El PCR puede detectar frecuencias de HHV- 8 amplificado. (Kevin R Flaherty, 2019)

Diagnóstico: Generalmente el diagnóstico es clínico, pero se deben tomar en cuenta la epidemiologia, ubicación geográfica, orientación sexual, es más frecuente en pacientes hombres que mantienes relaciones sexuales con hombres, presencia de lesiones mucocutáneas de KS, grado de 
inmunodeficiencia tomar en cuenta carga viral y CD4, presencia de lesiones radiológicas, lesiones a nivel bronquial y de haber descartado otros procesos infecciosos y neoplásicos. (Kevin R Flaherty, 2019)

Para establecer un diagnóstico de KS pulmonar se requiere de broncoscopia, para buscar lesiones endobronquiales típicas de KS y para excluir otras infecciones o patologías intercurrentes. El diagnostico se confirma con broncoscopia al identificar lesiones características de KS.

Diagnóstico diferencial: Los pacientes con SIDA pueden presentar sintomatología respiratoria debido a procesos infecciosos producidos por patógenos oportunistas y neoplasias asociadas tales como linfomas y enfermedad de Castleman multicéntrica. (Kevin R Flaherty, 2019)

Tratamiento: Tratamiento debes ser sistémico con terapia antiretroviral concomitante con quimioterapia local o sistémica.

\section{PRESENTACIÓN DE CASO CLÍNICO}

Paciente masculino de 22 años, sin antecedentes patológicos personales ni quirúrgicos de interés, como antecedentes familiares refiere que madre hace 1 año atrás presentó tuberculosis pulmonar cumpliendo esquema de tratamiento, alergias y hábitos tóxicos no refiere.

Paciente presentó cuadro clínico de aproximadamente un mes de evolución caracterizado por malestar general, presencia de pápulas en cara, tórax, miembros superiores e inferiores y pérdida de peso no cuantificada. A lo cual se suma sensación de alza térmica no cuantificada, tos de moderada intensidad con expectoración hemoptoica, una semana previa a su ingreso presentó un episodio de rectorragia.

Al examen físico se observa lesiones cutáneas papulosas induradas violáceas en extremidades superiores e inferiores, tórax y cara, además presentó inyección conjuntival derecha y hemorragia conjuntival izquierda. En mucosa oral presentaba lesiones rojo vinosas papulosas localizadas en paladar duro y blando, se palpan adenopatías cervicales e inguinales de 1 a $2 \mathrm{~cm}$ aproximadamente, no adheridas a planos profundos, no dolorosas. En campos pulmonares murmullo vesicular en los $2 / 3$ inferiores se encontraba disminuidos. Resto de examen físico sin patología aparente. 


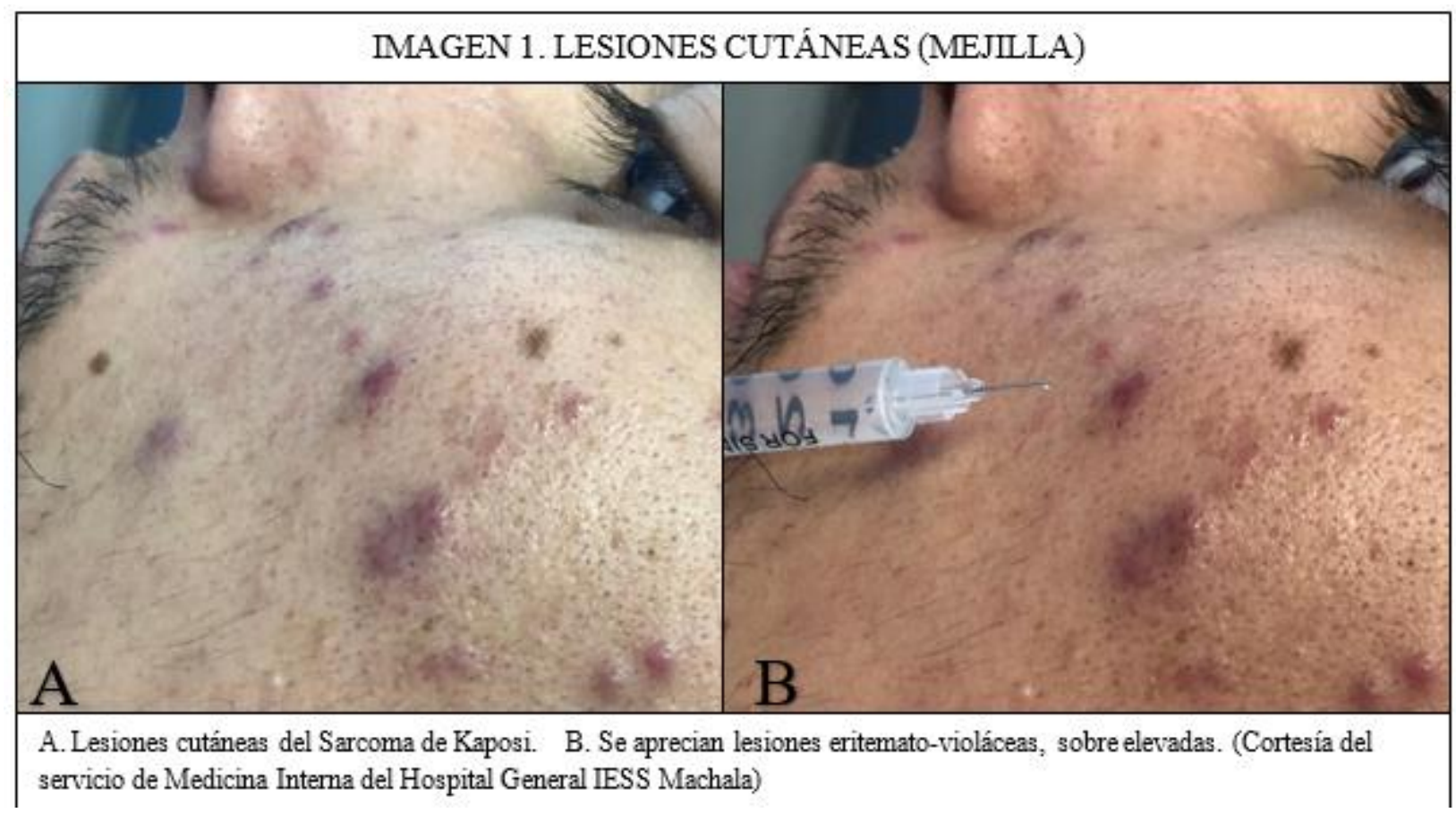

\begin{tabular}{|l|l|}
\hline \multicolumn{2}{|c|}{ TABLA 1. PARACLINICOS REALIZADOS } \\
\hline $\begin{array}{l}\text { Biometría hemática: } \\
\text { Anemia normocítica, normocrómica, trombocitopenia (54.000), linfocitos y neutrófilos dentro de } \\
\text { límites normales, tendiendo a la disminución. }\end{array}$ \\
\hline Química Sanguínea: & Dentro de parámetros normales. \\
\hline Coprológico, coproparasitario y EMO & Negativos \\
\hline Baciloscopias Seriadas & Negativas \\
\hline Pancultivos & Negativos \\
\hline $\begin{array}{l}\text { Serologías: } \\
\text { Herpes, Epstein Barr, Citomegalovirus, Hepatitis } \\
\text { A, B Y C }\end{array}$ & Negativos \\
\hline Anti-Toxoplasma IGM: & \\
\hline $\begin{array}{l}\text { Ensayo Inmunoenzimático De Cuarta } \\
\text { Generación: Elisa }\end{array}$ & Resitivo \\
\hline Cultivo del lavado bronquial & Negativo \\
\hline Genexpert & Negativo \\
\hline Recuentro de linfocitos CD4 & 12 cel/ul \\
\hline Carga viral & 219.000 \\
\hline $\begin{array}{l}\text { Resultado obtenidos de la base de datos de Laboratorio Clínico (Cortesía del servicio de Medicina } \\
\text { Interna del Hospital General IESS Machala) }\end{array}$ \\
\hline
\end{tabular}



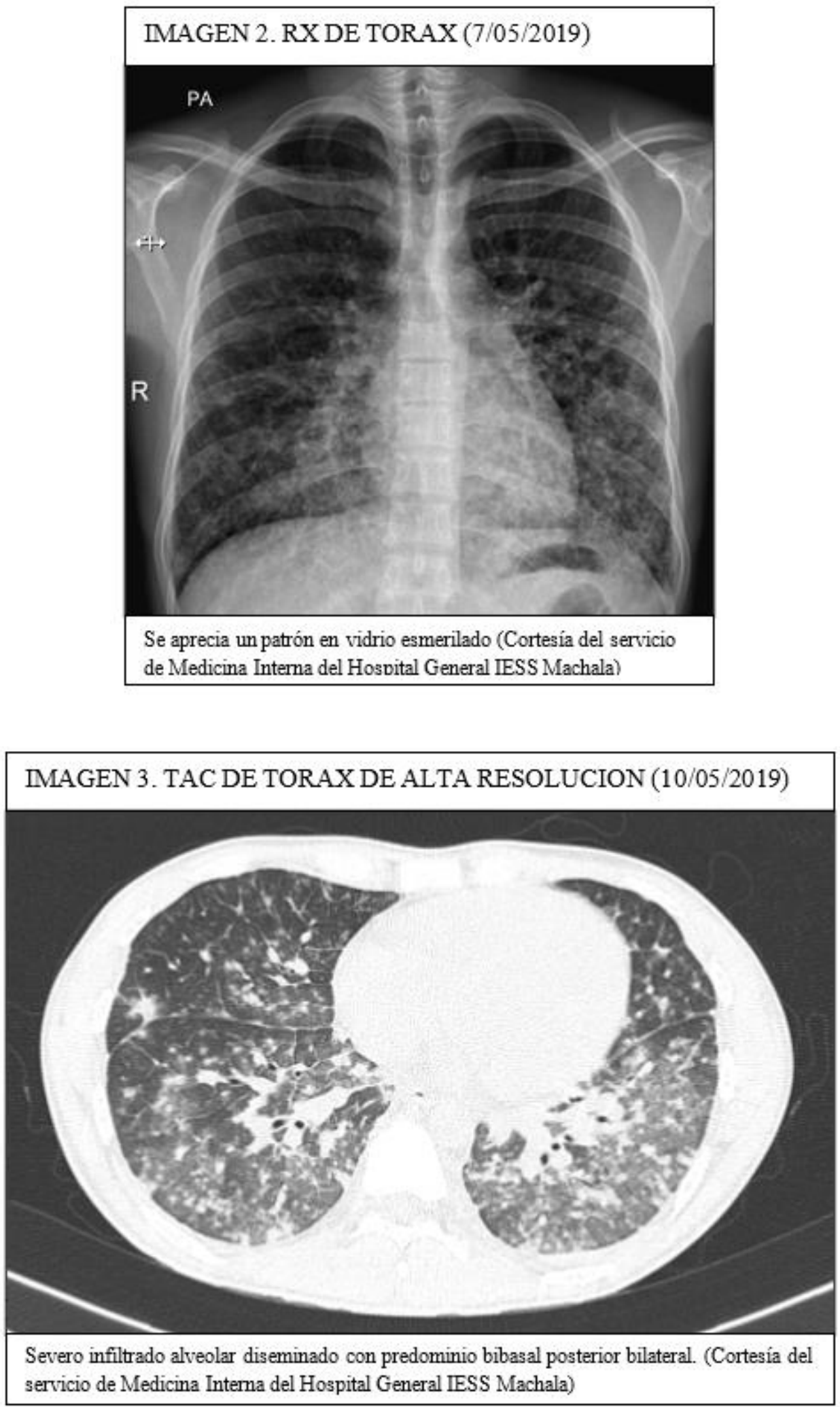


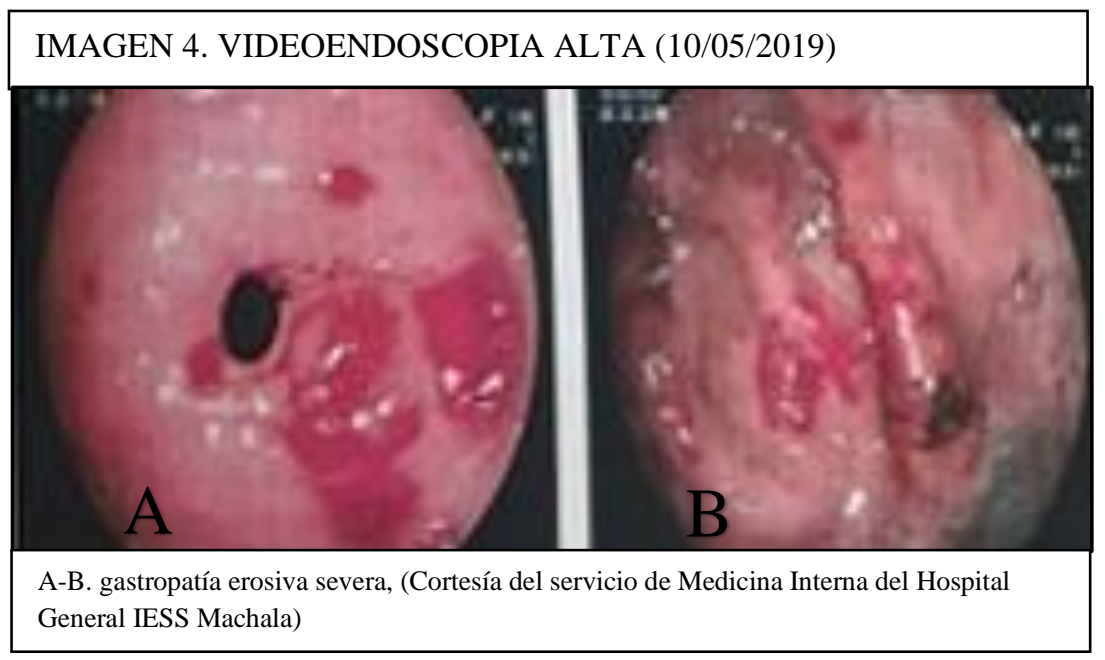

3 FIBROBRONCOSCOPIA:

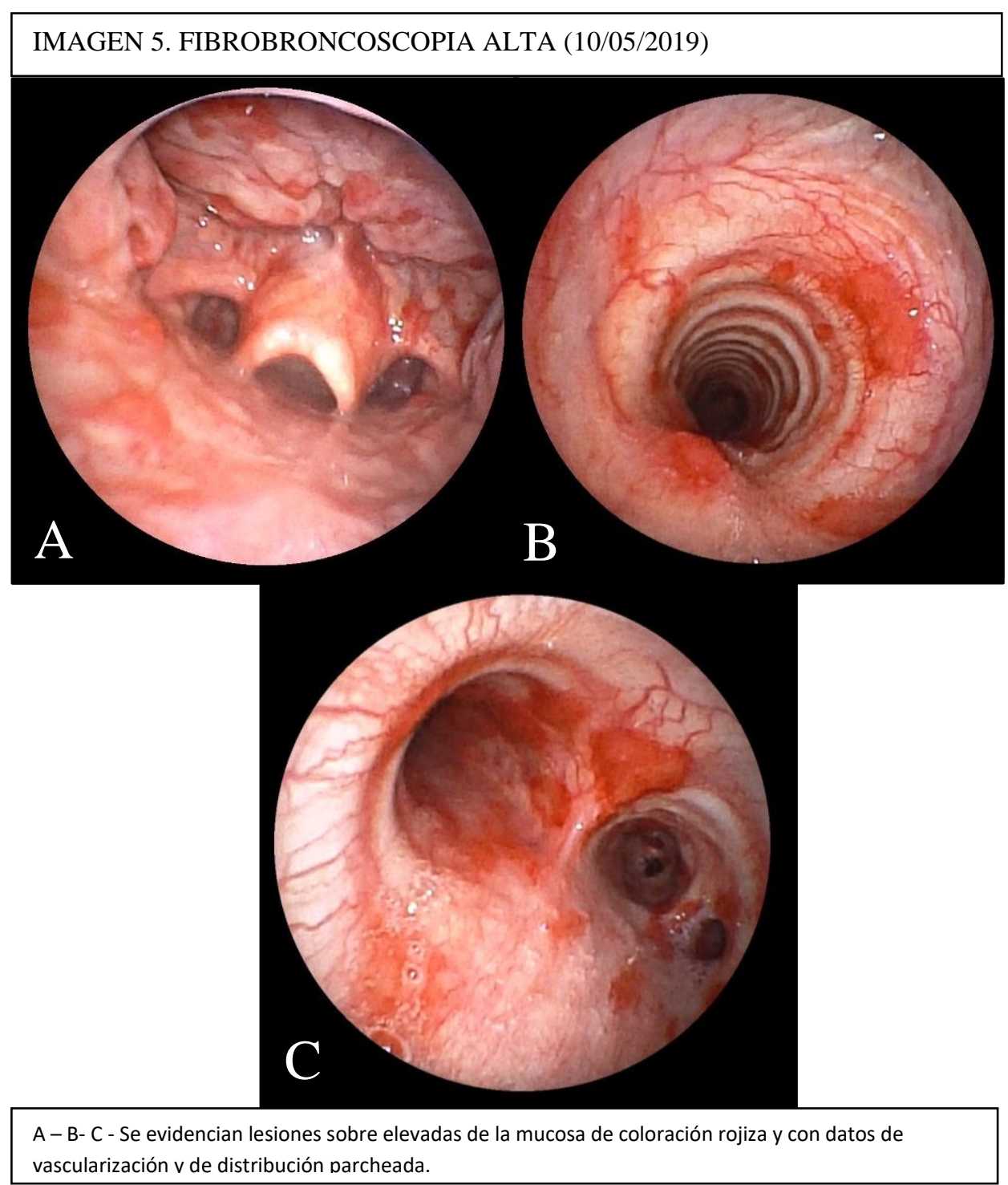




\section{BIOPSIA DE PIEL: EXAMEN MICROSCOPICO:}

Los cortes histológicos muestran estructura de piel revestida por epidermis irregular, a nivel de la dermis se observa proliferación de pequeños vasos sanguíneos, que forman hendiduras, revestidas por proliferación de células fusiformes, estrechamente adosadas entre sí, con núcleos ovoideos, hipercromáticos, con mitosis atípicas.

\section{Impresión diagnóstica: Sarcoma de Kaposi}

\section{DISCUSIÓN:}

El paciente durante su estancia hospitalaria presentó mejoría clínica respiratoria, las lesiones cutáneas papulosas violáceas han incrementado de tamaño. Se da alta hospitalaria con TARV

Sin embargo, 48 horas posteriores a su alta, paciente reingresa presentando mala mecánica ventilatoria (taquipnea, esfuerzo respiratorio). Con signos vitales PA: 100/60, FR: 28 con soporte de O2 por mascarilla con reservorio a 15 litros logrando saturación en 95\%. Al examen físico se auscultan roncus y estertores crepitantes predominantemente en campo pulmonar izquierdo.

Mantiene una mala evolución en cuanto su cuadro respiratorio, PAFI: 53 SDRA GRAVE, con mala respuesta a soporte ventilatorio, falleciendo en horas posteriores.

\section{CONCLUSIONES.}

Se conocen muy pocos casos de Sarcoma de Kaposi Pulmonar en pacientes con SIDA, ya que al no tener una sintomatología característica puede llegar a confundirse con otras enfermedades respiratorias prevalentes en pacientes inmunocomprometidos como tuberculosis pulmonar, histoplasmosis incluso otros tipos de neoplasias, es por eso que su diagnóstico constituye un gran reto, y en los pacientes en los que se puede llegar a un diagnóstico oportuno se debe iniciar inmediatamente tratamiento local y sistémico, a pesar de que la literatura menciona que no tiene un pronóstico bueno a corto plazo. 


\section{REFERENCIA BIBLIOGRAFICA}

- $\quad$ Enríquez., A. R. (enero-marzo 2013). Sarcoma de Kaposi: revisión de la literatura e ilustración de un caso. Acta Médica Grupo Ángeles. Volumen 11, No. 1,, 23:31.

- $\quad$ Haramati LB, W. J. (2000). Intrathoracic Kaposi's sarcoma in women with AIDS. Chest, 117:410.

- $\quad$ Hoskote SS, V. d. (2012). Sarcoma de Kaposi pulmonar en el SIDA. Mayo Clin Proc, 87: e77.

- JA., F.-S. (2015). Sarcoma de Kaposi asociado al VIH-SIDA. Artículo de revisión Rev Sanid Milit Mex, 476-482.

- Jiménez F, B. Y. (2011). Sarcoma de Kaposi gastrointestinal asociado a un síndrome de Inmunodeficiencia Adquirida. Descripción de un caso. . Acta Gastroenterológica Latinoamericana. , 308311. .

- Joshi M, M. N. (2006). Un paciente con VIH, disnea y múltiples nódulos pulmonares: sarcoma de Kaposi pulmonar. Cofre , 130: 1924.

- $\quad$ Kevin R Flaherty, M. M. (2019). Pulmonary involvement in AIDS-related Kaposi sarcoma. Uptodate. , 1:19.

- $\quad$ Lodi S, G. M. (2010). Kaposi sarcoma incidence and survival among HIV-infected homosexual men after HIV seroconversion. J Natl Cancer Inst , 102:784.

- Nathan S, V. R. (1990). Uso de láser Nd: YAG en el sarcoma endobronquial de Kaposi. Pecho, 98: 1299.

- Onak Kandemir N, B. F. (2013). Cavernous Hemangioma-Like Kaposi Sarcoma: Histomorphologic Features and Differential Diagnosis. Case Reports in Medicine.

- $\quad$ P Cacopardo B, i. M. (2015). virus de Nunnari G. Epstein-barr y malignidades relacionadas con el virus del herpes asociado al sarcoma de Kaposi en el contexto de la infección por el virus de la inmunodeficiencia humana. Semin Oncol, 42: 25.

- $\quad$ Palmieri C. et al. (2006). Pulmonary Kaposi's sarcoma in the era of highly active antiretroviral therapy. HIV Med 7:, $291-292$.

- Yoo DJ, L. K. (2005). Hallazgos clínicos y broncoscópicos en ugandeses con sarcoma de Kaposi pulmonar. Coreano J Intern Med, 20: 290. 